\title{
Coordinated inventory replenishment and outsourced transportation operations
}

\author{
Ülkü Gürler ${ }^{\mathrm{a}}$, Osman Alp ${ }^{\mathrm{b}, *}$, Nasuh Çağdaş Büyükkaramikli ${ }^{\mathrm{c}}$ \\ ${ }^{a}$ Department of Industrial Engineering, Bilkent University, 06800 Ankara, Turkey \\ ${ }^{\mathrm{b}}$ Department of Industrial Engineering, TED University, Kolej, 06420 Ankara, Turkey \\ ${ }^{\mathrm{c}}$ Erasmus University Rotterdam, Institute of Health Policy and Management, P.O. Box 1738, 3000DR Rotterdam, The Netherlands
}

\section{A R T I C L E I N F O}

\section{Article history:}

Received 17 December 2013

Received in revised form 9 July 2014

Accepted 13 August 2014

\section{Keywords:}

Third party logistics

Inventory

Transportation

Coordinated logistics

\begin{abstract}
A B S T R A C T
We consider a one-warehouse $N$ retailers supply chain with stochastic demand. Inventory is managed in-house whereas transportation is outsourced to a 3PL provider. We develop analytical expressions for the operating characteristics under both periodic and continuous joint replenishment policies. We identify the settings where a periodic review policy is comparable to a continuous review one. In our numerical test-bed, the periodic policy performed best in larger supply chains operating with larger trucks. We also observed that if the excess utilization charge is less than $25 \%$, outsourcing becomes beneficial even if outsourcing cost is $25 \%$ more than the in-house fleet costs.
\end{abstract}

(c) 2014 Elsevier Ltd. All rights reserved.

\section{Introduction}

Inventory management and transportation are among the major operations of supply chains. In practice, these operations are generally managed in a decoupled fashion. Approximately 1.2 trillion USD or about $8.5 \%$ of the US. GDP is attributed to the total logistics activities in USA in 2011 (Burnson, 2012). Of these logistics costs, transportation (largely trucking costs) accounted for $63 \%$ while inventory carrying costs accounted for $33 \%$ in the US economy in 2002 (FHWA, 2005). A recent study by Buyukkaramikli et al. (2014) reveals the value of coordinating these two logistics operations in a particular setting in which retail orders are centrally shipped by an in-house fleet of vehicles from a warehouse or a distributor to multiple retailers. We extend this model to consider the case where the transportation operations are outsourced to a third party logistics (3PL) provider through a transportation contract. In particular, we consider a one-warehouse- $N$-retailer supply chain where inventory replenishment decisions at the retailers are made in a coordinated fashion and the retail orders are shipped by a third party carrier with their own fleet of vehicles according to the terms of a contract. Firms started to use outsourcing as a strategic management tool since 1990s and have been outsourcing some of their key business operations including logistics since then (Craumer, 2002). As mentioned by Lieb and Bentz (2005), outsourcing logistics functions has been favorable among large US manufacturers not just to control costs but also as a means of differentiation in domestic and international markets.

3PL companies provide a broad collection of logistics activities including freight forwarding, consolidation centers, direct transportation service, inventory management/replenishment, warehouse management, in-store logistics, delivery services, and reverse logistics (see Ton and Wheelwright, 2005, for a full list). The market for 3PL providers increases gradually in

\footnotetext{
* Corresponding author. Tel.: +90 3125850023.

E-mail addresses: ulku@bilkent.edu.tr (Ü. Gürler), osman.alp@tedu.edu.tr (O. Alp), buyukkaramikli@bmg.eur.nl (N.Çağdaş Büyükkaramikli).
} 
years; the gross revenue of the global market was 391 Billion USD in 2007 (Lin and Yen, 2008) whereas this figure jumped to 616.1 Billion USD in 2011 (Langley and Capgemini, 2013). The results of the annual 2013 3PL survey show the size and the benefits of outsourcing logistics, based on more than 2000 companies captured worldwide by the survey: (i) firms that use 3PL providers spend about $12 \%$ of their revenues to logistics costs whereas $39 \%$ of this cost is spent for outsourced logistics activities, and (ii) outsourcing accounted for a $15 \%$ cost reduction in logistics operations on the average for all companies worldwide. Even though 3PL providers offer a wide range of logistics activities to their customers, the "transactional, operational, and repetitive" activities, such as transportation, are the ones that are outsourced the most; whereas "more strategic, customer facing and IT-intensive" activities, such as inventory management, order management and fulfillment are less frequently outsourced (Langley and Capgemini, 2013). Fig. 1 compares the worldwide percentages of logistics activities outsourced and percentages of 3PL companies that provide such services.

In this study, we consider a firm which prefers to outsource only the transportation operation and runs the inventory management in-house. Economies of scale, savings in capital investments, and reduction in financial risks are among the advantages of using 3PL providers for transportation (Vasiliauskas and Jakubauskas, 2007). Other advantages are increasing efficiency, controlling costs, and reliability and speed (Ton and Wheelwright, 2005). The continuous growth in the 3PL market lead to consolidation in 3PL industry, and large 3PL companies have emerged consequently (Selviaridis and Spring, 2007). For example, Giraud Logistics acquired four transportation operators from Belgium, France, Spain and Italy between the years 1999 and 2001 (Carbone and Stone, 2005), which further was acquired by Geodis Logistics in the year 2010 (EUbusiness, 2010). Such giant logistics companies serve to a wide collection of customers including shippers, receivers, and carriers. These are either "asset-based" or "non-asset-based" companies depending on whether they own their fleet or not. Non-asset-based companies find transportation capacity from the market whenever necessary. Asset-based 3PL companies also can find additional transportation capacity easily from the market whenever necessary (Applegate, 2002). Therefore, one can safely assume that 3PL providers do not experience any constraint on the number of trucks that they can provide to their customers, in general.

There are substantial number of papers in literature that deal with different aspects of 3PL transportation. For example, Alp et al. (2003) deal with an inventory control problem under deterministic demand and stochastic lead time where transportation is conducted by a 3PL provider. Cai et al. (2013) consider the pricing decision of a 3PL provider and its impact on the performance of a supply chain where a producer ships fresh products to a downstream distributor which resells the product to end customers. Li et al. (2008) deal with the problem of coordinating 3PL transportation with manufacturing scheduling in a make-to-order consumer electronics supply chain. Ulku and Bookbinder (2012) deal with the price and delivery time quotation decisions of a 3PL provider in a price and time sensitive logistics market for a long range haul between an origin and destination.

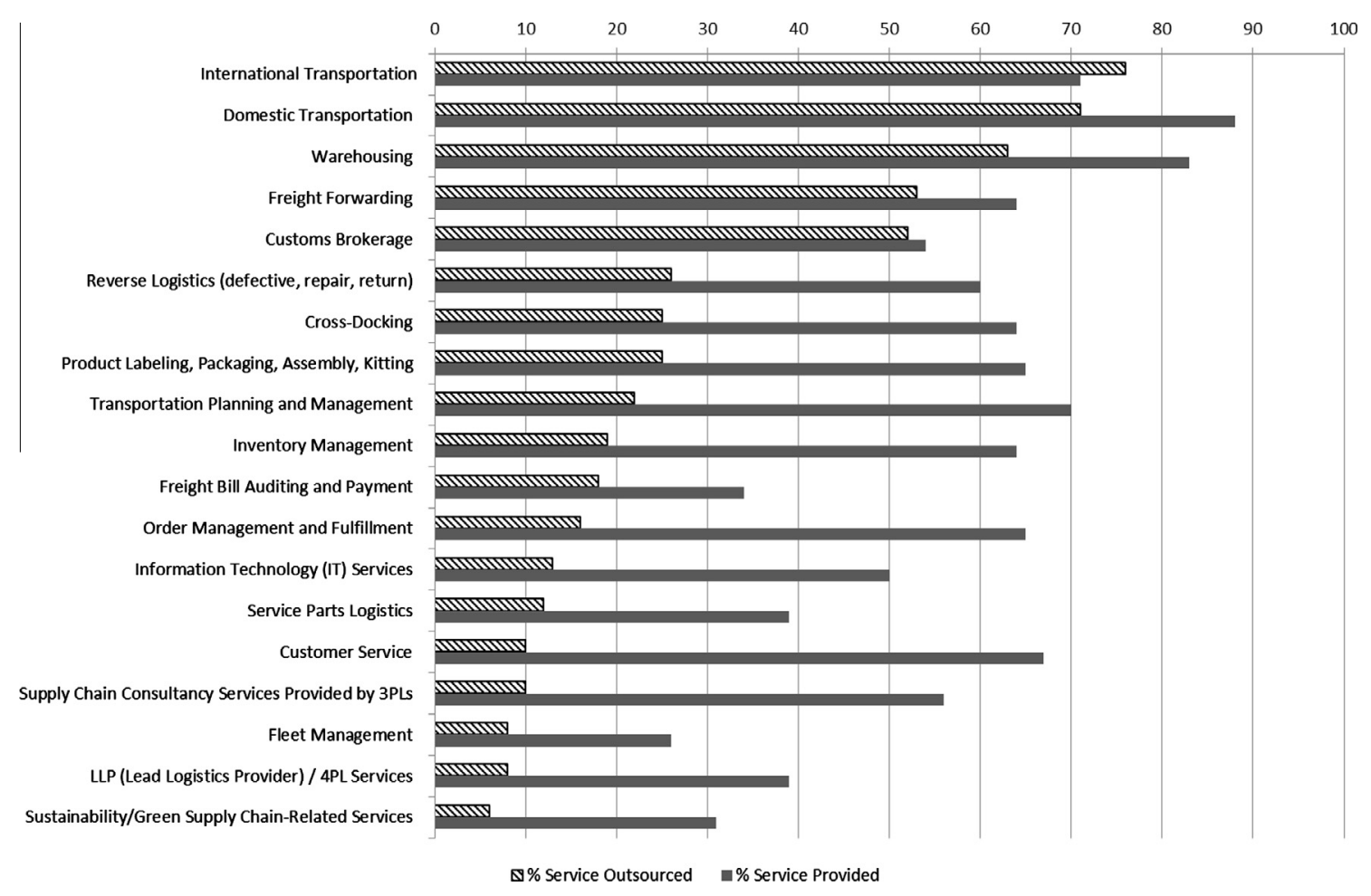

Fig. 1. Percentage of logistics activities outsourced vs provided by 3PL companies (source: Langley and Capgemini, 2013). 
Another relevant stream of research in literature is related to integrated logistics, where in particular inventory and transportation operations are considered simultaneously. Archetti et al. (2011) discuss such a model in a deterministic demand setting with a single depot, multiple retailers, and a fixed fleet of vehicles. The authors show that when the transportation is outsourced and the retailers use an order-up-to policy, the problem is NP-hard. The studies by Berman and Wang (2006), Kang and Kim (2010), and Konur and Schaefer (2014) are other examples that assume deterministic demand and integrate the costs of inventory and transportation operations. Similar problems with stochastic demand are also considered by several authors. In this setting, Kutanoglu and Lohiya (2008) consider a service parts logistics system in a single echelon setting with time-based service level constraints. They aim to optimize inventory control policies together with transportation mode selection, alternatives being slow, medium, and fast transportation. Cachon (2001) and Tanrikulu et al. (2010) analyze a supply chain environment where the joint replenishment orders of retailers are dispatched by an ample supplier with capacitated trucks of an unlimited fleet. Gurbuz et al. (2007) assume that the joint orders are shipped by a single truck from the warehouse to a cross-dock facility and if the order quantity exceeds the truck capacity, it is still shipped with an additional penalty cost. Madadi et al. (2010) analyze centralized and decentralized integrated decisions of inventory replenishment and transportation operations with unlimited fleet in a periodic review setting with normal demand. In a recent study Buyukkaramikli et al. (2014) consider an integrated inventory and transportation problem of a two echelon inventory system with one warehouse and multiple retailers where the outbound shipments are handled by a limited size in-house fleet of vehicles under a continuous review operation environment. A vast number of research in the context of integrated inventory replenishment and dispatching can be found in vendor managed inventory models (see e.g. Mutlu and Cetinkaya, 2010 and Zhao et al., 2010) in which the problem setting and the modeling and analysis methodology are quite different than our focus.

In this work, we consider an integrated model for inventory replenishment and transportation where the transportation operations are outsourced to a 3PL provider firm, under two joint replenishment policies for the retailers, namely the $(Q, S)$ and $(\mathbf{R}, T)$ policies. We consider a risk neutral approach, hence our aim is to minimize the long-run expected cost rate function with respect to policy parameters. Our work provides both an analytical modeling approach for integrating inventory and transportation operations, and managerial insights for the pros and cons of adapting alternative joint replenishment policies when transportation activities are outsourced to a 3PL firm. In-house versus outsourcing strategies are also compared under the $(Q, S)$ replenishment policy. Specifically, in this study we

(i) analytically derive the operating characteristics for the integrated joint inventory replenishment and outsourced transportation models for two different replenishment policies;

(ii) derive the service level expressions for both replenishment policies where the service level expression for the $(Q, S)$ policy is not provided before in literature to the best of our knowledge;

(iii) identify operational scenarios where a periodic review policy (which is preferred in practice due to ease of implementation) would be a cost-wise preferred or reasonable choice over a continuous review policy; and

(iv) identify the operating characteristics where a 3PL transportation strategy surpasses an in-house fleet transportation strategy.

Similar to Cachon (2001) and Tanrikulu et al. (2010), we assume that the 3PL carrier can provide unlimited number of trucks if and whenever necessary. However, different from their work, we allow for 3PL contracts that involve cost structures which charge higher unit prices beyond some pre-specified usage. Our work differs from Buyukkaramikli et al. (2014) in that (i) we consider an outsourcing option for the transportation, whereas they assume a limited size in-house fleet and (ii) in addition to the $(Q, S)$ policy employed therein, we also consider the $(\mathbf{R}, T)$ policy, details of which are given below. We compare the two transportation strategies and two joint replenishment policies under different problem settings.

The rest of the paper is organized as follows. In Section 2, we provide the operations environment and the model assumptions. In Sections 3 and 4, we analyze the continuous review $(Q, S)$ and the periodic review $(\mathbf{R}, T)$ policy, respectively. Comparison of alternative inventory policies and transportation strategies are provided in Section 5. Finally, we discuss our results and present our conclusions in Section 6.

\section{Environment and model assumptions}

We consider a centrally controlled, divergent two-echelon supply chain with a single warehouse and $N$ retailers. Retailers face stochastic customer demand and their orders are replenished by the warehouse. A joint replenishment protocol is implemented to manage the inventories of the retailers. We consider two such policies, one is the continuous review $(Q, S)$ policy and the other is the periodic review $(\mathbf{R}, T)$ policy. The central authority implements a cross-dock distribution strategy where the joint orders destined to replenish the retailers are streamed to the cross-dock station first, and are dissipated to each individual retailer thereafter with smaller trucks (see Fig. 2). The notation used is introduced in the text whenever necessary and is also summarized in Table 1.

In the $(Q, S)$ policy, a joint order of size $Q$ is placed to raise the inventory positions of items to their order-up-to levels $\mathbf{S}=\left(S_{1}, S_{2}, \ldots, S_{N}\right)$ when a total demand of $Q$ units accumulate for all items. Pantumsinchai (1992) presents an exact analysis of this policy under Poisson demands. This policy is considered by Cheung and Lee (2002) in a two echelon setting. Atkins 


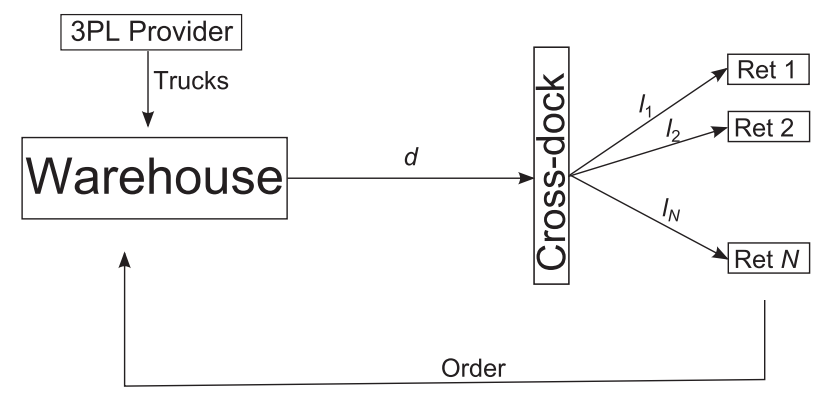

Fig. 2. Illustration of the environment.

Table 1

Summary of notation used.

\begin{tabular}{ll}
\hline$N$ & Number of retailer \\
$\lambda_{i}$ & The rate of Poisson demand at retailer $i$ \\
$\lambda_{0}$ & Total demand rate at all retailers, i.e. $\sum_{i=1}^{N} \lambda_{i}$ \\
$F_{p}(y \mid \lambda)$ & Cumulative Poisson probability with rate $\lambda$ \\
$L_{i}$ & Replenishment lead time at retailer $i$ \\
$C$ & Truck size \\
$K$ & (reserved) Fleet size \\
$D$ & Total occupation time of a truck before it can be available for a new shipment \\
$h_{i}$ & Unit holding cost per unit time \\
$b_{i}$ & Unit backordering cost per unit time \\
$A$ & Fixed cost of shipment \\
$\phi(K)$ & Cost of maintaining/reserving a fleet of $K$ trucks \\
$\alpha$ & Outsourcing penalty \\
$\beta$ & Excess usage penalty \\
$Q$ & Order size in $(Q, S)$ policy \\
$S_{i}$ & Order-up-to level of retailer $i$ in $(Q, S)$ policy \\
$T$ & Order intervals in $(\mathbf{R}, T)$ policy \\
$R_{i}$ & Order-up-to level in $(\mathbf{R}, T)$ policy \\
\hline
\end{tabular}

and Iyogun (1988) propose the $(\mathbf{R}, T)$ policy for the joint replenishment problem, where this policy dictates to raise the inventory positions of the items at every $T$ periods up to their corresponding order-up-to levels $\mathbf{R}=\left(R_{1}, R_{2}, \ldots, R_{N}\right)$. The authors compare the performance of this policy to that of the can-order policy, and show that $(\mathbf{R}, T)$ policy outperforms the latter in many instances.

The major transportation operation in this chain, namely the transportation of the joint orders from the warehouse to the cross-dock station, is outsourced to a 3PL provider which we refer to as the carrier. A transportation contract which specifies the services required and the costs in lieu, is signed between the carrier and the supply chain authority. The carrier guarantees to allocate as many trucks as necessary at any point in time to ship the orders from the warehouse to the cross-dock station without any delays. However, their truck resources are not unlimited as they would be doing business with several other customers at the same time. Therefore, the carrier agrees to "reserve" a given number of trucks, say $K$, for a base price; but charges extra costs for excess usage, if necessary. Consequently, we consider an "options contract" where the carrier charges (i) a fixed cost of $\phi(K)$ per unit time where $K$ is the base number of trucks reserved by the carrier and (ii) an options cost of $\beta \frac{\phi(K)}{K}$ per truck whenever the average number of trucks utilized per unit time exceeds $K$ trucks, where $\beta$ is a contract parameter which is greater than one. We consider $\beta$ as the excess usage penalty, larger values of which will make 3PL contract less beneficial. As mentioned above, Buyukkaramikli et al. (2014) consider a similar problem with in-house (IH) fleet transportation. The cost of reserving $K$ trucks under 3PL outsourcing is expected to be higher than the cost of maintaining an inhouse fleet of $K$ trucks. For comparison of the present work with their results, we account for this by letting the fleet maintenance cost in IH be $\phi(K) / \alpha$ for some $\alpha \geqslant 1$. Here $\alpha$ is considered as an outsourcing penalty, the value of which is external, ie. determined by the particular business, as opposed to the excess utilization cost which is a contract parameter. Regarding the truck capacities, we assume that all trucks allocated by the carrier has the same size, $C$. The contract also specifies a duration, $D$, through which each truck used for a shipment is regarded as "occupied with this shipment". Note that $D \geqslant d$ where $d$ is the one-way trip duration between the warehouse and the cross-dock station. $D$ might be strictly greater than $d$ as it can include a return trip to the warehouse or an additional time required for the truck to be re-used for another shipment by the carrier. We assume that the total time $D$ on transit of a vehicle is constant. In practice, the time on transit may be random due to several effects such as traffic load or immediate unavailability of vehicles. If the possible variability on transportation time is small relative to $D$, then constancy may be a safe assumption. Note however that the "reliability" of the transit times is one of the key concerns of the logistics decision makers because it has a significant adverse effect on inventory operations 
(Dullaert and Zamparini, 2013). Hence if the uncertainty in transportation times is not negligible, other modeling approaches which may lead to non-trivial problems should be adopted as discussed at the end of our work.

The retailers face stationary and independent unit Poisson demands with rates $\lambda_{i},(i=1,2, \ldots, N)$, and unmet demands are fully backordered. The total demand rate for the retailers is given by $\lambda_{0}=\sum_{i=1}^{N} \lambda_{i}$. Holding and shortage costs incurred at retailer $i$ are denoted by $h_{i}$ and $b_{i}$, respectively, per unit per time. We assume that the warehouse is an ample supplier. Replenishment lead time of retailer $i$ is $L_{i}=d+l_{i}$ (see Fig. 2). For each truck utilized for shipment, a fixed cost of $A$ which is independent of the quantity loaded incurs. Some examples are the fuel costs of transportation, costs of driver and the dispatcher, or fixed administrative ordering costs. Depending on the negotiated contractual terms, this fixed cost of ordering might be undertaken by the carrier or might be incurred to the supply chain authority explicitly. For the sake of generality, we include this term in our cost function. Consequently, the total operating costs in this chain consists of the expected inventory holding and backorder costs at the retailers, fixed costs associated with an order in each truck, and transportation costs dictated by the contract.

\section{Continuous inventory replenishment: $(Q, S)$ policy}

In this section we consider the continuous review $(Q, \mathbf{S})$ policy which is implemented to manage the inventories of the retailers. This policy dictates to raise the inventory position of each retailer up to their corresponding order-up-to level $\mathbf{S}=\left(S_{1}, S_{2}, \ldots, S_{n}\right)$ whenever a total of $Q$ units are demanded in the entire chain. Note here that since we assume unit demands and continuous review, $Q$ in our settings corresponds to both the replenishment threshold and the order size.

\subsection{Inventory related costs}

The total expected inventory holding and backordering cost at retailer $i$ is given by

$$
g_{i}\left(S_{i}\right)=\frac{1}{\lambda_{i}}\left[S_{i}\left(h_{i}+b_{i}\right) F_{P}\left(S_{i} \mid \lambda_{i} L_{i}\right)-\lambda_{i} L_{i}\left(h_{i}+b_{i}\right) F_{P}\left(S_{i}-1 \mid \lambda_{i} L_{i}\right)+b_{i}\left(\lambda_{i} L_{i}-S_{i}\right)\right]
$$

where $F_{P}(y \mid \lambda)$ denotes the cumulative probability distribution of a Poisson variable with rate $\lambda$. Hence, the total inventory holding and backordering costs in this supply chain can be written as follows:

$$
U_{q s}(Q, S, K)=\sum_{i=1}^{N} \lambda_{i} \frac{1}{Q} \sum_{n=0}^{Q-1} \sum_{m_{i}=0}^{n}\left(\begin{array}{c}
n \\
m_{i}
\end{array}\right)\left(\lambda_{i} / \lambda_{0}\right)^{m_{i}}\left(1-\lambda_{i} / \lambda_{0}\right)^{n-m_{i}} g_{i}\left(S_{i}-m_{i}\right) .
$$

The fixed costs of ordering per unit time is given by $\lambda_{0} \frac{A}{Q}$. We refer the reader to Buyukkaramikli et al. (2014) for the details of derivations of these functions.

\subsection{Transportation costs}

Let $B$ denote the random number of busy trucks at any time. Since the number of busy trucks depend on the inventory dispatching system and there is no limit on the number of trucks provided by the carrier, $B$ is a random variable that takes nonnegative integer values. Also, recall that under the $(Q, S)$ policy, each retailer demand is relayed as an individual order and waits until a total of $Q$ individual orders are accumulated to form a joint order. Let $W$ denote the random variable corresponding to the total number of individual demands accumulated at the retailers at any time. Then, $W$ can take any integer value between 0 and $Q-1$. The time between orders is Erlang distributed with shape parameter $Q$ and $W=j$ corresponds to the $j$ th exponential stage of this Erlang distribution.

This stochastic system can be characterized by a two dimensional state variable $(b, w)$ where $b$ and $w$ are realizations of $B$ and $W$, respectively. We define $P_{t}\{(b, w)\}$ as the transient probability that the system is at state $(b, w)$ at time $t$. Similarly, we let $P\{(b, w)\}=\lim _{t \rightarrow \infty} P_{t}\{(b, w)\}$ be the stationary joint probability distribution of $B$ and $W$. Then we have the following result:

Theorem 1.

$$
P\{(b, w)\}=\left\{\begin{array}{ll}
\sum_{i=0}^{w} \frac{1}{Q} F_{P}\left(w-i \mid \lambda_{0} D\right) & \text { for } b=0,0 \leqslant w \leqslant Q-1 \\
\sum_{i=0}^{Q-1} \frac{1}{Q} F_{P}\left(b Q+w-i \mid \lambda_{0} D\right) & \text { for } b \geqslant 1,0 \leqslant w \leqslant Q-1
\end{array} .\right.
$$

Proof. See Appendix.

Let $B_{q s}^{K+}$ be the random variable denoting the number of busy trucks above $K$ at any given time under $(Q, S)$ policy. Then its expectation is:

$$
E\left[B_{q s}^{K+}\right]=\sum_{b=K+1}^{\infty} \sum_{w=0}^{Q-1}(b-K) P\{(b, w)\} .
$$




\subsection{Total expected operating costs}

Consequently, the total expected operating costs can be written as

$$
A C_{q s}(Q, S, K)=\lambda_{0} \frac{A}{Q}+\phi(K)+\beta \frac{\phi(K)}{K} E\left[B_{q s}^{K+}\right]+U_{q s}(Q, S, K) .
$$

Theorem 2. Let $\phi(K)=a K$ where $a>0$. Then, $A C_{q s}(Q, S, K)$ is convex in $K$.

Proof. See Appendix.

\subsection{Service levels}

When minimizing the expected cost rate of inventory systems with backordering, either a backorder cost is incurred when an arriving demand is not immediately satisfied, or a service level constraint is imposed if the backorder cost is hard to estimate. The two options can be made equivalent by the proper choices of the backorder cost and the service level. In our model, we assume a positive backorder cost and optimize the system without the service level constraint. However it is also interesting to know the attained service levels under the optimal system parameters. Below we provide the results for assessing the service levels of the system under the $(Q, S)$ replenishment policy. First we need to characterize the steady state probability distribution of the inventory levels of each retailer at an arbitrary time instance. To this end, we first let $\left(K_{i}(t), J_{i}(t)\right), t \geqslant 0$ denote a stochastic process with state space $S S=\{(k, j), k=0,1, \ldots, Q-1 ; j=0,1, \ldots, Q-1\}$, where $K(t)$ and $J(t)$ refer to the total demand observed at retailer $i$ and the total demand observed at all retailers other than $i$, respectively at time $t$, since the last order placement instance. Then, $S_{i}-K(t)$ denotes the inventory position of retailer $i$ at time $t$. Due to the nature of the $(Q, S)$ policy, $K(t)+J(t) \leqslant Q-1$ since whenever $K(t)+J(t)=Q$, a replenishment order is placed and the inventory position of retailer $i$ is raised to $S_{i}$ (i.e. $K(t)$ becomes 0 ) and $J(t)$ drops to 0 . Consequently, the inventory system of retailer $i$ can be characterized by the state pair $\left(S_{i}-K(t), J(t)\right)$. The corresponding state transition diagram is depicted in Fig. 5 in Appendix.

Theorem 3. Let $I P_{i}$ be the random variable denoting the inventory position of retailer $i$. The probability distribution of $I P_{i}$ at the steady state of the system is as follows:

$$
P\left\{I P_{i}=S_{i}-k\right\}=\sum_{j=0}^{Q-1-k}\left(\begin{array}{c}
j+k \\
j
\end{array}\right)\left(\frac{\lambda_{i}}{\lambda_{0}}\right)^{k}\left(1-\frac{\lambda_{i}}{\lambda_{0}}\right)^{j} \frac{1}{Q} \quad \text { for } k \in\{0,1, \ldots, Q-1\}
$$

Proof. See Appendix.

Theorem 4. Let $I L_{i}$ be the random variable denoting the inventory level of retailer $i$. The probability distribution of $I L_{i}$ at the steady state of the system is as follows:

$$
P\left\{I L_{i}=x\right\}=\sum_{k=0}^{Q-1} P\left\{I P_{i}=S_{i}-k\right\} f_{P}\left(S_{i}-k-x \mid \lambda_{i} L_{i}\right) \quad \text { for } x \in\left(-\infty, S_{i}\right]
$$

where $f_{p}\left(S_{i}-k-x \mid \lambda_{i} L_{i}\right)$ is the probability distribution function of a Poisson variable which corresponds to observing $S_{i}-k-x$ units of demand at retailer $i$ during its lead time, $L_{i}$.

Proof. See Appendix.

Finally, we can state the service level attained at Retailer $i$ under the $(Q, S)$ policy as

$$
S L_{Q S}^{i}=P\left\{I L_{i} \geqslant 0\right\}
$$

\section{Periodic inventory replenishment: $(R, T)$ policy}

In this section we assume that the retailers are replenished by the periodic review $(\mathbf{R}, T)$ policy. Periodic review policies are practically appealing because of their ease of implementation and synchronization with the upper stream. Under this policy, the system is reviewed on a periodic basis, with period length $T$ and the inventory positions of the retailers are raised up to $\mathbf{R}$, the vector of order-up-to levels, at each review epoch. The order quantity placed at the end of a review period is a random variable, corresponding to the total demand faced by the retailer during that period. Since each retailer observes unit 
Poisson demands, the order quantity, $X$, is Poisson with an expected value of $\lambda_{0} T$. This implies that the number of trucks needed to dispatch a given order is also a random variable.

\subsection{Inventory related costs}

The expected total inventory holding and backordering costs of the entire chain can be written as follows (Hadley and Whitin, 1963):

$$
U_{r t}(\mathbf{R}, T, K)=\sum_{i=1}^{n} h_{i}\left(R_{i}-\lambda_{i} L_{i}-\frac{\lambda_{i} T}{2}\right)+\left(h_{i}+\beta_{i}\right) \Pi\left(R_{i}, T\right)
$$

where

$$
\begin{aligned}
\Pi\left(R_{i}, T\right)= & \frac{1}{T}\left\{\frac{\lambda_{i}}{2}\left[\left(L_{i}+T\right)^{2} \bar{F}_{P}\left(R_{i}-1 \mid \lambda\left(L_{i}+T\right)\right)-L_{i}^{2} \bar{F}_{P}\left(R_{i}-1 \mid \lambda_{i} L_{i}\right)\right]+\frac{R_{i}\left(R_{i}+1\right)}{2 \lambda_{i}}\left[\bar{F}_{P}\left(R_{i}+1 \mid \lambda_{i}\left(L_{i}+T\right)\right)\right.\right. \\
& \left.\left.-\bar{F}_{P}\left(R_{i}+1 \mid \lambda_{i} L_{i}\right)\right]-R_{i}\left[\left(L_{i}+T\right) \bar{F}_{P}\left(R_{i} \mid \lambda_{i}\left(L_{i}+T\right)\right)-L_{i} \bar{F}_{P}\left(R_{i} \mid \lambda_{i} L_{i}\right)\right]\right\}
\end{aligned}
$$

and $\bar{F}_{P}(y \mid \lambda)=1-F_{P}(y-1 \mid \lambda)$. Expected fixed cost of ordering is given by $A \frac{E[B]}{T}$ where $B$ is the random variable denoting the number of busy trucks at any time.

\subsection{Transportation costs}

Let $B_{r t}(\tau)$ denote the random variable corresponding to the total number of trucks shipped at the last $\tau$ review periods under $(\mathbf{R}, T)$ policy, i.e. $B_{r t}(\tau)=\sum_{i=1}^{\tau}\left\lceil\frac{X}{C}\right\rceil$. The following recursive relation can be used to estimate the probability distribution of $B_{r t}(\tau)$ for all integers $i \geqslant 0$ :

$$
\begin{aligned}
& P\left\{B_{r t}(1)=i\right\}=P\{(i-1) C<X<i C\} \\
& P\left\{B_{r t}(\tau)=i\right\}=\sum_{k=0}^{i} P\left\{B_{r t}(1)=k\right\} P\left\{B_{r t}(\tau-1)=i-k\right\} \quad \text { for } \tau>1
\end{aligned}
$$

Theorem 5. The expected average number of busy trucks above the reserved quantity K during any review cycle is given by

$$
E\left[B_{r t}^{K+}\right]=\frac{E\left[\left(B_{r t}(\eta+1)-K\right)^{+}\right](D-\eta T)+E\left[\left(B_{r t}(\eta)-K\right)^{+}\right]((\eta+1) T-D)}{T}
$$

where $\eta=\left\lfloor\frac{D}{T}\right\rfloor$ and $E\left[\left(B_{r t}(\eta)-K\right)^{+}\right]=\sum_{i=K+1}^{\infty}(i-K) P\left\{B_{r t}(\eta)=i\right\}$.

Proof. See Appendix.

Consequently, the total cost rate per unit time in this policy is given by

$$
A C_{r t}(\mathbf{R}, T, K)=A \frac{E[B]}{T}+\phi(K)+\beta \frac{\phi(K)}{K} E\left[B_{r t}^{K+}\right]+U_{r t}(\mathbf{R}, T, K) .
$$

Theorem 6. Let $\phi(K)=a K$ where $a>0$. Then $A C_{r t}(\mathbf{R}, T, K)$ is convex in $K$.

Proof. See Appendix.

\subsection{Service levels}

For the periodic review (R, $T$ ) policy, the service level can be measured by the probability that no stock-outs are experienced during one replenishment cycle. Suppose that an order is placed for retailer $i$ at an order period $t$. All outstanding orders at time $t$ will be received before $t+L_{i}$ and the order placed at time $t$ will be received at time $t+L_{i}$. Since an order is placed every $T$ time periods, no other replenishment can occur during $\left(t+L_{i}, t+L_{i}+T\right)$. Therefore, Retailer $i$ can observe a stock-out situation during $\left[t+L_{i}, t+L_{i}+T\right)$ only if the total demand during $L_{i}+T$ units of time exceeds $R_{i}$. Consequently, we can express the service level attained at Retailer $i$ as

$$
S L_{R S}^{i}=F_{p}\left(R_{i} \mid \lambda_{i}\left(L_{i}+T\right)\right)
$$

which corresponds to the probability of observing at most $R_{i}$ units of demand in $L_{i}+T$ units of time. 


\section{Comparison of alternative inventory and transportation operations}

In this section, we provide our numerical findings regarding (i) the relative performances of alternative inventory replenishment policies when transportation operation is outsourced and (ii) relative costs of alternative transportation options given as in-house or outsourcing, under the $(Q, S)$ replenishment policy. Regarding the replenishment policies, it is generally known that continuous review policies outperform the periodic ones since it allows to trace all the changes in system states instantaneously. Hence a priori, one would expect that the continuous review $(Q, S)$ policy would dominate the periodic review $(\mathbf{R}, T)$ policy. Our numerical results indeed supports this expectation, as they are along this line in a vast majority of the problem instances (see Section 5.1). However, practicality of a sub-optimal policy may be the main advantage over a better or an optimal policy for their employment in real life. This is one of the reasons why periodic review policies are so commonly used by the firms. We identify the operational characteristics where the periodic review policy either outperforms the continuous review policy or produces cost terms slightly higher. From the transportation point of view, it is interesting to find out the advantages and disadvantages of in-house versus outsourcing strategies, as this is a relatively less studied issue in the integrated management of transportation and replenishment functions (see Section 5.2). Results for the in-house transportation under the $(Q, S)$ policy was given in Buyukkaramikli et al. (2014). Operational characteristics of the outsourcing option derived in this study allowed us to compare the relative performances of such options.

The primary parameters of the integrated replenishment and transportation supply chain model studied herein are the size $N$ of the supply chain, the total demand arrival rate $\lambda_{0}$, the two-way transportation time $D$, the truck capacity $C$ and the inventory holding and backorder costs $h, b$ respectively. In the choice of experimental set, we aimed to select the values of these parameters that would allow us (i) to compare our findings with the available results in literature and (ii) to gain insights for a reasonably wide range of settings. Hence referring to Cachon (2001) and Buyukkaramikli et al. (2014) we considered the following set: $N \in\{2,8,16\}, \lambda_{0} \in\{8,16,32,64\}, D \in\{2,4,8\} C \in\{4,8,12,16\}$. For the inventory costs, as commonly assumed we set $h=1$, and let the backorder cost change as $b \in\{4,8,16\}$. The retailers are assumed to be identical in their demand rate, holding and backordering costs, and lead times. We assumed negligible time $\left(l_{i}=0\right)$ from the cross-dock to the retailers, so that $L_{i}=D / 2$. Even though the fixed cost of shipment, $A$, does not depend on the quantity loaded in the vehicle, it might depend on the size of the truck. Hence, we consider four different truck sizes, which directly imply four different fixed costs. We set this fixed cost function as a concave function, $A=C^{\gamma}$ where $0<\gamma \leqslant 1$. Further parameter values specific to a particular transportation alternative will be given in the related section.

\section{1. $(Q, \mathbf{S})$ vs $(\mathbf{R}, T)$ policies under Outsourced Transportation}

In this section, we compare the performances of $(Q, S)$ and $(\mathbf{R}, T)$ policies. In order to measure the relative cost difference we define

$$
\Delta_{R T}=\frac{A C_{r t}^{*}-A C_{q s}^{*}}{A C_{q s}^{*}} \times 100
$$

where $A C_{r t}^{*}$ and $A C_{q s}^{*}$ are as defined in Eqs. (1) and (2). We set $A(C)=C^{0.25}$.

We first note that in almost all of the 1296 problem instances in our test bed, $(Q, S)$ policy outperforms $(\mathbf{R}, T)$ policy in terms of total expected costs. $(\mathbf{R}, T)$ policy yields a lower cost in only six of the instances. The minimum, maximum, and average $\Delta_{R T}$ values are $-1.59,20.39$, and 9.14, respectively. In Table 2, we present the average relative performance of the $(\mathbf{R}, T)$ policy for all combinations of $\lambda, N$ and $C$ values. We observe that the performance of the $(\mathbf{R}, T)$ policy gets closer to that of the $(Q, S)$ policy for lower demand rates, higher truck capacities and larger number of retailers. Consequently, all six cases with a negative $\Delta_{R T}$ value lie in this extreme of the parameter values: $\lambda=8, C=16$ and $N=16$. In all other combinations of $\lambda, C$, and $N$, the average $\Delta_{R T}$ values are greater than $2 \%$, pointing out that the $(Q, S)$ policy dominates the $(\mathbf{R}, T)$ policy by a significant amount considering the scale of big businesses. Finally, we note that the advantage gap of the $(Q, S)$ policy reduces for high demand rates accompanied with lower capacity trucks or low demand rates with higher capacity trucks for a given $N$ value.

Tables 3-5 present the relative cost performance for a subset of problem instances considered. There is no monotonic relation between each problem parameter and the relative performance of the $(\mathbf{R}, T)$ policy. Problem parameters have a close interaction among each other so that finding the parameter ranges where $(\mathbf{R}, T)$ performs relatively better is not possible. For the problem instances with lower $\Delta_{R T}$ values, $(\mathbf{R}, T)$ tends to perform better in terms of inventory holding and backordering

Table 2

Average $\Delta_{R T}$ values for all $b, D$, and $\beta$.

\begin{tabular}{|c|c|c|c|c|c|c|c|c|c|c|c|c|}
\hline \multirow[t]{2}{*}{$\lambda$} & \multicolumn{3}{|l|}{$C=4$} & \multicolumn{3}{|l|}{$C=8$} & \multicolumn{3}{|l|}{$C=12$} & \multicolumn{3}{|c|}{$C=16$} \\
\hline & $N=2$ & $N=8$ & $N=16$ & $N=2$ & $N=8$ & $N=16$ & $N=2$ & $N=8$ & $N=16$ & $N=2$ & $N=8$ & $N=16$ \\
\hline 8 & 15.08 & 9.51 & 7.65 & 15.44 & 7.73 & 5.46 & 10.35 & 4.34 & 2.91 & 6.92 & 2.71 & 1.14 \\
\hline 16 & 13.10 & 8.98 & 7.31 & 16.86 & 10.06 & 7.33 & 14.38 & 7.48 & 5.31 & 10.14 & 5.10 & 3.27 \\
\hline 32 & 10.69 & 7.74 & 6.27 & 15.84 & 10.42 & 7.97 & 16.83 & 9.75 & 7.65 & 14.29 & 8.28 & 5.77 \\
\hline 64 & 8.31 & 6.25 & 5.20 & 13.46 & 9.08 & 7.40 & 16.27 & 10.17 & 8.43 & 16.41 & 10.00 & 7.66 \\
\hline
\end{tabular}


Table 3

Impact of backordering cost on average $\Delta_{R T}$ when $D=4$ and $\beta=1.25$.

\begin{tabular}{|c|c|c|c|c|c|c|c|c|c|c|c|c|}
\hline \multirow[t]{3}{*}{$\lambda$} & \multicolumn{6}{|l|}{$N=2$} & \multicolumn{6}{|c|}{$N=16$} \\
\hline & \multicolumn{3}{|l|}{$C=4$} & \multicolumn{3}{|l|}{$C=16$} & \multicolumn{3}{|l|}{$C=4$} & \multicolumn{3}{|l|}{$C=16$} \\
\hline & $b=4$ & $b=8$ & $b=16$ & $b=4$ & $b=8$ & $b=16$ & $b=4$ & $b=8$ & $b=16$ & $b=4$ & $b=8$ & $b=16$ \\
\hline 8 & 16.07 & 15.11 & 14.42 & 7.11 & 6.41 & 6.55 & 8.60 & 8.80 & 6.68 & 1.80 & 1.65 & 1.58 \\
\hline 16 & 13.52 & 13.21 & 12.58 & 10.69 & 9.25 & 8.45 & 9.83 & 7.52 & 4.95 & 4.59 & 2.05 & 2.26 \\
\hline 32 & 10.49 & 10.49 & 10.45 & 15.67 & 13.50 & 12.30 & 6.51 & 5.55 & 5.02 & 6.60 & 5.45 & 4.74 \\
\hline 64 & 8.18 & 8.30 & 8.30 & 17.66 & 17.01 & 16.17 & 5.13 & 5.10 & 5.28 & 8.92 & 8.17 & 6.92 \\
\hline
\end{tabular}

Table 4

Impact of trucking time on average $\Delta_{R T}$ when $b=8$ and $\beta=1.25$

\begin{tabular}{|c|c|c|c|c|c|c|c|c|c|c|c|c|}
\hline \multirow[t]{3}{*}{$\lambda$} & \multicolumn{6}{|l|}{$N=2$} & \multicolumn{6}{|c|}{$N=16$} \\
\hline & \multicolumn{3}{|l|}{$C=4$} & \multicolumn{3}{|l|}{$C=16$} & \multicolumn{3}{|l|}{$C=4$} & \multicolumn{3}{|c|}{$C=16$} \\
\hline & $D=2$ & $D=4$ & $D=8$ & $D=2$ & $D=4$ & $D=8$ & $D=2$ & $D=4$ & $D=8$ & $D=2$ & $D=4$ & $D=8$ \\
\hline 8 & 17.72 & 15.11 & 12.70 & 10.08 & 9.14 & 11.45 & 5.90 & 8.80 & 6.73 & 2.58 & 1.65 & 1.63 \\
\hline 16 & 16.28 & 13.21 & 10.33 & 12.80 & 13.63 & 15.18 & 9.52 & 7.52 & 5.13 & 2.80 & 2.05 & 4.35 \\
\hline 32 & 13.66 & 10.49 & 8.29 & 16.90 & 17.57 & 14.99 & 8.56 & 5.55 & 4.72 & 3.37 & 5.45 & 6.75 \\
\hline 64 & 10.52 & 8.30 & 6.33 & 19.46 & 15.87 & 13.29 & 6.26 & 5.10 & 4.15 & 7.45 & 8.17 & 6.32 \\
\hline
\end{tabular}

Table 5

Impact of excess usage penalty on average $\Delta_{R T}$ when $b=8$ and $D=4$

\begin{tabular}{|c|c|c|c|c|c|c|c|c|c|c|c|c|}
\hline \multirow[t]{3}{*}{$\lambda$} & \multicolumn{6}{|l|}{$N=2$} & \multicolumn{6}{|l|}{$N=16$} \\
\hline & \multicolumn{3}{|l|}{$C=4$} & \multicolumn{3}{|l|}{$C=16$} & \multicolumn{3}{|l|}{$C=4$} & \multicolumn{3}{|l|}{$C=16$} \\
\hline & $\beta=1.25$ & $\beta=2$ & $\beta=5$ & $\beta=1.25$ & $\beta=2$ & $\beta=5$ & $\beta=1.25$ & $\beta=2$ & $\beta=5$ & $\beta=1.25$ & $\beta=2$ & $\beta=5$ \\
\hline 8 & 15.11 & 15.09 & 14.79 & 9.14 & 8.83 & 10.27 & 8.80 & 8.86 & 8.79 & 1.65 & 1.32 & 1.72 \\
\hline 16 & 13.21 & 13.21 & 13.00 & 13.63 & 14.05 & 15.05 & 7.52 & 7.55 & 7.50 & 2.05 & 2.02 & 2.03 \\
\hline 32 & 10.49 & 10.42 & 10.26 & 17.57 & 18.03 & 17.98 & 5.55 & 5.55 & 5.51 & 5.45 & 5.68 & 5.89 \\
\hline 64 & 8.30 & 8.38 & 8.31 & 15.87 & 16.15 & 16.13 & 5.10 & 5.09 & 5.08 & 8.17 & 8.34 & 8.50 \\
\hline
\end{tabular}

cost and extra truck usage cost. However, those savings are dominated by the increase in ordering and truck reservation costs. The problem instance with $N=16, \lambda=8, b=16, C=16, D=2$, and $\beta=5$ is the instance which yields the minimum $\Delta_{R T}$ value in our test bed. Further details of our observations are provided in the tables and the discussion below.

Table 3 presents the cost differences for three levels of backorder costs, two levels of truck capacities, and two levels of the number of retailers. We observe here that the cost differences are not very sensitive to the backorder costs but truck capacity and demand rate are more influential, with an obvious interaction of truck capacity and demand rate. In particular, when the truck capacity is low, the cost differences decrease as the demand rate increases and it changes in just the opposite direction when truck capacity is high. Since the order quantities are directly related and limited by the truck capacities, higher $C$ values indicate higher $Q$ values as well. This results in higher cost for the $(\mathbf{R}, T)$ policy for high demand rates most probably due to higher delay costs.

In Table 4, we again observe a similar interaction with truck capacity and demand rate. As $D / 2$ corresponds to the retailer lead time, we see that if lead time demand is high and truck capacity (correspondingly $Q$ ) is low, or if the lead time demand is low but the truck capacity is high, the disadvantage of $(\mathbf{R}, T)$ policy reduces. Roughly, we observe that under a periodic review policy, truck capacity should be inversely proportional to the demand rate. Table 5 presents the impact of excess usage penalty on the average cost differences. Here, we again note that the truck size and the demand rate have more significant impact than the excess usage cost, with similar implications of the two previous tables.

Table 6 presents the optimal $K$ values under both policies and some other policy parameters. In general, we observe that the number of trucks reserved in $(\mathbf{R}, T)$ policy is higher than that in $(Q, S)$ policy, and this pays off better in higher demands for smaller trucks and vice versa for the larger trucks. We also present the service levels attained by each policy for the problem instances depicted in Fig. 6. We observe that when $b=16$, the service levels range between 93-98\%. If such service levels are not acceptable for the decision maker, higher values of $b$ should be selected.

As a final note, we observe that in all the tables (except Table 6) $\Delta_{R T}$ values are positive, advocating the $(Q, S)$ policy. However, our finding is that in general these differences get smaller for big supply chains with many retailers, with high transportation capacities and low demand rates. In particular we observe that for $N=16$ and $C=16$ and $\lambda=8$, smallest differences are observed. Furthermore, in Table 6 , we note that $\Delta_{R T}$ changes from -1.6 to 1.0 when $\lambda$ changes from 8 to 16 when $D=2, C=16$. This indicates that for some intermediate value of $\lambda$ between 8 and 16 the difference will attain a value zero. Hence there is a non empty set of parameters where these two policies are cost equivalent. 
Table 6

Comparison of the policies when $N=16, b=16$ and $\beta=5$.

\begin{tabular}{|c|c|c|c|c|c|c|c|c|c|c|c|c|c|c|c|}
\hline \multirow[t]{3}{*}{$D$} & \multirow[t]{3}{*}{$\lambda$} & \multicolumn{7}{|c|}{$C=4$} & \multicolumn{7}{|c|}{$C=16$} \\
\hline & & \multicolumn{3}{|l|}{$(\mathbf{R}, T)$} & \multicolumn{3}{|c|}{$(Q, \mathbf{S})$} & \multirow[b]{2}{*}{$\Delta_{R T}$} & \multicolumn{3}{|l|}{$(\mathbf{R}, T)$} & \multicolumn{3}{|c|}{$(Q, S)$} & \multirow[b]{2}{*}{$\Delta_{R T}$} \\
\hline & & $T^{*}$ & $K^{*}$ & $S L$ & $Q^{*}$ & $K^{*}$ & $S L$ & & $T^{*}$ & $K^{*}$ & $S L$ & $Q^{*}$ & $K^{*}$ & $S L$ & \\
\hline \multirow[t]{3}{*}{2} & 8 & 0.4 & 7 & 0.97 & 4 & 5 & 0.98 & 7.6 & 0.7 & 3 & 0.95 & 9 & 2 & 0.96 & -1.6 \\
\hline & 16 & 0.3 & 12 & 0.96 & 4 & 9 & 0.97 & 8.3 & 0.5 & 4 & 0.93 & 9 & 4 & 0.96 & 1.0 \\
\hline & 32 & 0.25 & 21 & 0.96 & 4 & 18 & 0.98 & 6.0 & 0.35 & 7 & 0.94 & 15 & 5 & 0.96 & 3.7 \\
\hline \multirow[t]{3}{*}{8} & 8 & 1 & 21 & 0.96 & 4 & 18 & 0.98 & 4.4 & 1.15 & 7 & 0.95 & 14 & 5 & 0.96 & 1.6 \\
\hline & 16 & 0.8 & 38 & 0.94 & 4 & 34 & 0.94 & 4.6 & 0.8 & 12 & 0.94 & 16 & 9 & 0.96 & 4.2 \\
\hline & 32 & 0.4 & 75 & 0.95 & 4 & 67 & 0.96 & 4.9 & 0.4 & 24 & 0.94 & 16 & 17 & 0.95 & 6.1 \\
\hline
\end{tabular}

SL: service level (probability of no stock-out).

\subsection{Outsourcing vs. in-house transportation under the $(Q, S)$ policy}

We considered in this work an integrated inventory and transportation model where the dispatching of the orders to the retailers is outsourced to a 3PL provider. As discussed earlier, this is one of the employed business strategies in practice. We denote this strategy as '3PL option'. An alternative to outsourcing is to keep an in-house fleet for order deliveries. In this system, transportation operations are managed centrally by the supply chain authority along with the inventory operations. We denote this strategy as 'IH option'. The central authority owns a limited fleet of vehicles to transport the joint orders placed by the retailers. The fleet size is denoted by $K$ and the capacity of each vehicle is denoted by $C$, as in the outsourcing case. Unlike the outsourcing case, in-house fleet transportation may result in delays in the shipments of the orders from the warehouse to the retailers due to vehicle shortages at the base. In such cases, orders are delayed, bringing extra inventory shortage costs. Such delays are not allowed in the 3PL option since the service provider supplies extra vehicles whenever necessary, albeit at an extra cost. Buyukkaramikli et al. (2014) analyze the IH option and derives expected overall total costs of operations when the retailers are managed by the $(Q, S)$ policy. In this section, we utilize those results to compare the performances of these two transportation systems. Due to the differences between the IH and 3PL options in terms of shipment delays discussed above, orders never wait at the warehouse for shipment under 3PL. Hence, the expected inventory related costs at the retailers is always less under the 3PL option. On the other hand, elevated transportation costs are incurred with 3PL provider if the expected number of busy trucks exceeds the reserved number of trucks of size $K$. As discussed previously, we let the fleet maintenance cost in IH be $\phi(K) / \alpha$ where $\phi(K)$ is the reservation cost of $K$ trucks of capacity $C$ under 3PL and $\alpha>1$ is the outsourcing penalty. We assume that the fixed ordering and fleet related costs are functions of the vehicle capacity, $C$, and use the concave functions $A=C^{0.9}$ and $\phi(K)=K\left(1-C^{-0.5}\right)$ in our numerical study. Finally, we note that we make the performance comparison based on the operational costs in the following discussion, and ignore the fixed capital costs of the assets under IH option. However, this cost component can easily be incorporated in the fleet cost function, $\phi(K) / \alpha$, by considering the investment costs and useful lives of the vehicles.

Let $\Delta_{T C}$ be the percentage relative difference between the optimal expected costs of 3PL and IH. In particular,

$$
\Delta_{T C}=\frac{\left(A C_{3 P L}^{*}-A C_{I H}^{*}\right)}{\min \left(A C_{3 P L}^{*}, A C_{I H}^{*}\right)} \times 100
$$

where $A C^{*}$ denotes the corresponding optimal cost. Hence a negative value of $\Delta_{T C}$ indicates that $3 \mathrm{PL}$ is less costly than IH option, and vice versa. Similarly, percentage relative differences of individual cost components are denoted by $\Delta_{i c}, \Delta_{o c}$, and $\Delta_{s c}$ for inventory, ordering, and shipment costs, respectively. We refer to all transportation related costs other than the fixed ordering costs as the shipment cost. Finally, $\Delta_{k}$ denotes the difference between the optimal number of reserved trucks in 3PL and the optimal fleet size in IH. A negative value indicates that more trucks are reserved in IH.

In Table 7, we report a comparison of 3PL and IH for a number of problem instances. For all of these cases $\Delta_{o c}=0$, hence are not reported and $\Delta_{i c}$, is sensitive only to the changes in $N$ and $D$. When the outsourcing penalty $(\alpha)$, the excess utilization penalty $(\beta)$, total tome on-transit $(D)$ of trucks and the size of the supply chain $(N)$ are relatively low, outsourcing results in lower costs, as the values of these parameters increase the negative differences turn to have positive values. Hence we note that there would be a sizable set of parameter values where $\Delta_{T C}$ will assume value zero and the two transportation options would be equivalent. This table also provides the components of the total expected cost rate. We observe that for large values of $\alpha$ and $\beta$, shipment costs of 3PL increases and IH becomes the preferred option. The table further shows that the number of trucks reserved in outsourcing is generally less than that in IH transportation. This makes sense since in IH-transportation there is no option to handle emergency cases, a larger fleet size would be held to hedge for the delay risks. However, as the excess usage penalty, $\beta$, increases the gap between in-house fleet size and the reserved number of trucks in 3PL narrow down. We observe that $\Delta_{k}$ values are non-decreasing in $\beta$ and non-increasing in $\alpha$, as expected. The relative characteristics of both strategies remain similar when $N$ is increased to 16 . However, relative differences in the total expected costs decreases in general when $N$ is increased from 2 to 16 . In the table we see that the inventory related costs are not effected 
Table 7

Comparison of IH and 3PL when $\lambda=16, b=4, C=4$.

\begin{tabular}{|c|c|c|c|c|c|c|c|c|c|c|}
\hline \multirow[t]{2}{*}{$N$} & \multirow[t]{2}{*}{$\alpha$} & \multirow[t]{2}{*}{$\beta$} & \multicolumn{4}{|l|}{$D=2$} & \multicolumn{4}{|l|}{$D=8$} \\
\hline & & & $\Delta_{T C}$ & $\Delta_{i c}$ & $\Delta_{s c}$ & $\Delta_{k}$ & $\Delta_{T C}$ & $\Delta_{i c}$ & $\Delta_{s c}$ & $\Delta_{k}$ \\
\hline \multirow[t]{4}{*}{2} & 1 & 1.25 & -3.94 & -3.47 & -17.84 & -3 & -4.02 & -2.30 & -9.13 & -6 \\
\hline & & 2 & -2.71 & & -9.58 & -2 & -2.63 & & -5.09 & -4 \\
\hline & & 5 & -1.01 & & 0.37 & -1 & -0.74 & & 0.11 & -2 \\
\hline & & 10 & -0.04 & & 5.82 & 0 & 0.34 & & 3.02 & 0 \\
\hline \multirow[t]{4}{*}{2} & 1.2 & 1.25 & -1.54 & -3.47 & -2.55 & -4 & 2.00 & -2.30 & 7.5 & -9 \\
\hline & & 2 & 0.24 & & 7.25 & -2 & 3.93 & & 12.74 & -5 \\
\hline & & 5 & 2.24 & & 18.37 & -1 & 6.25 & & 19 & -2 \\
\hline & & 10 & 3.58 & & 25.82 & 0 & 7.63 & & 22.76 & -1 \\
\hline \multirow[t]{4}{*}{2} & 1.5 & 1.25 & -0.62 & -3.47 & 2.5 & -9 & 3.61 & -2.30 & 11.87 & -35 \\
\hline & & 2 & 4.11 & & 28.78 & -3 & 13.37 & & 38.28 & -6 \\
\hline & & 5 & 7.10 & & 45.37 & -1 & 16.69 & & 47.27 & -3 \\
\hline & & 10 & 8.97 & & 55.75 & -1 & 18.41 & & 51.92 & -1 \\
\hline \multirow[t]{4}{*}{16} & 1 & 1.25 & -2.27 & -0.88 & -17.84 & -3 & -2.05 & -1.25 & -6.10 & -5 \\
\hline & & 2 & -1.51 & & -9.58 & -2 & -1.23 & & -2.17 & -3 \\
\hline & & 5 & -0.46 & & 0.37 & -1 & -0.09 & & 2.97 & -1 \\
\hline & & 10 & 0.16 & & 5.82 & 0 & 0.56 & & 5.97 & 1 \\
\hline \multirow[t]{4}{*}{16} & 1.2 & 1.25 & -0.79 & -0.88 & -2.55 & -4 & 1.57 & -1.25 & 10.57 & -8 \\
\hline & & 2 & 0.33 & & 7.25 & -2 & 2.75 & & 15.96 & -4 \\
\hline & & 5 & 1.59 & & 18.37 & -1 & 4.16 & & 22.40 & -1 \\
\hline & & 10 & 2.43 & & 25.82 & 0 & 5.01 & & 26.27 & 0 \\
\hline \multirow[t]{4}{*}{16} & 1.5 & 1.25 & -0.21 & -0.88 & 2.5 & -9 & 2.56 & -1.25 & 15.07 & -34 \\
\hline & & 2 & 2.77 & & 28.78 & -3 & 8.51 & & 42.23 & -5 \\
\hline & & 5 & 4.65 & & 45.37 & -1 & 10.54 & & 51.48 & -2 \\
\hline & & 10 & 5.83 & & 55.75 & -1 & 11.59 & & 56.27 & 0 \\
\hline
\end{tabular}

by the excess usage and outsourcing penalties, hence only a single value appears. Regarding the retailer lead time $D$, we observe different features for $D=2$ and $D=8$ cases. In particular, when the in-house transportation is more beneficial (positive values in the table), relative advantage is larger for longer travel distance. That is, for smaller distances the loss due to transferring the transportation function to a 3PL provider becomes less. In almost all cases (except $N=2, \alpha=1, \beta=1.25$ ), inhouse transportation is more beneficial with respect to the total cost.

Fig. 3 shows the change in $\Delta_{T C}$ for different $\lambda$ and $b$ values when $\alpha=1.2$ and $\beta=2$, where $\Delta_{T C}$ values are the averages over all values of $C$ and $D$ values. We observe that $\Delta_{T C}$ increases with the demand, meaning that IH option becomes more attractive, since the need for (and the cost of) extra trucks in 3PL increases as $\lambda$ increases. For higher values of $b$, 3PL option becomes more attractive since the main advantage of 3PL is to keep backorder costs under control by utilizing extra trucks whenever necessary. Hence, we can conclude that IH would be a preferable option over 3PL under high demand rates and low backordering costs.

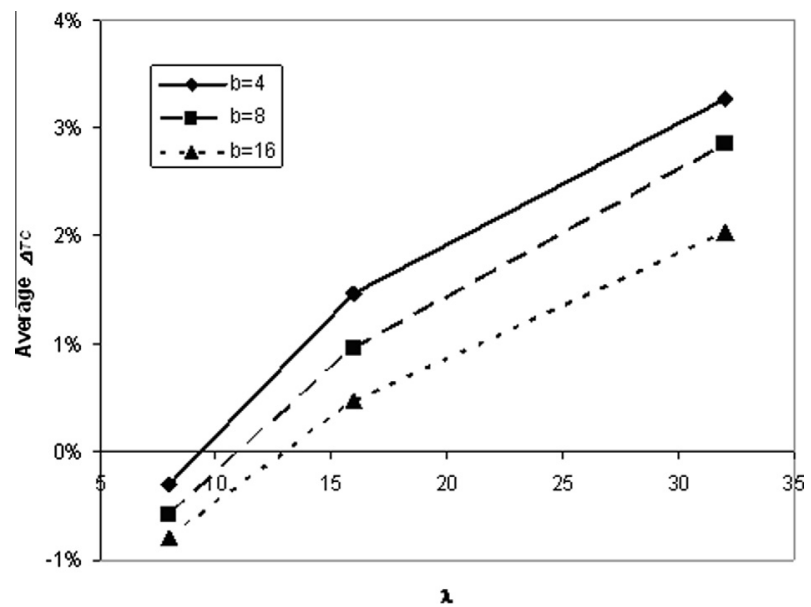

Fig. 3. Demand rate vs. average cost deviation for different backordering costs when $\alpha=1.2$ and $\beta=2$. 


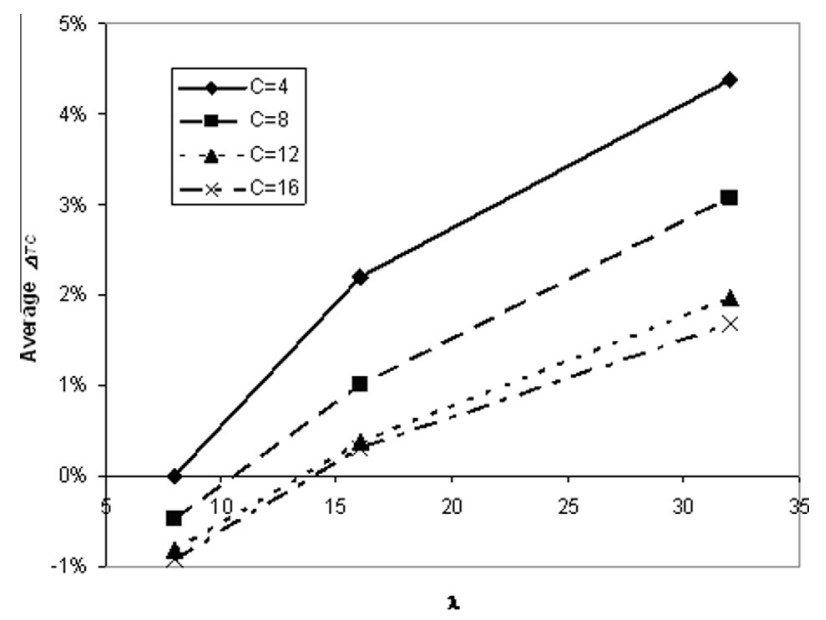

Fig. 4. Demand rate vs. average cost deviation for different truck capacities when $\alpha=1.2$ and $\beta=2$.

Fig. 4 shows the change in $\Delta_{T C}$ for different $\lambda$ and $C$ values when $\alpha=1.2$ and $\beta=2$, where the $\Delta_{T C}$ are the averages over all values of $b$ and $D$ in the test bed. In this figure, we observe that outsourcing gains advantage as the truck capacity increases. This is not an obvious result since increased truck capacity is beneficial to both IH (as it reduces the adverse effect of limited size) and to 3PL provider (as it implies less frequent usage of extra trucks). We observe in general that as $C$ increases, (i) total inventory cost of IH converges to that of outsourcing, (ii) the fixed ordering cost increases, leading to larger batch sizes and reduced ordering costs in both models, and (iii) outsourcing experiences larger savings on shipments costs. This saving seems to dominate the relative changes in other cost components and therefore 3PL becomes more advantageous as $C$ increases in general. Finally, we note that the relative differences in $\Delta_{T C}$ decreases as $C$ increases because of the concave nature of the transportation related costs with respect to $C$.

\section{Conclusions and discussions}

In this study, we consider an integrated model for the inventory replenishment and transportation operations of a supply chain with $N$ retailers and a single warehouse, where the inventory operations are managed in-house but the transportation operation is outsourced. This particular management model of transportation and inventory operations is of significant practical relevance as evidenced by surveys in the literature. In particular, surveys conducted in the last decade about 3PL market reveal that, while majority of the firms prefer to outsource their transportation operations, most of them have in-house management of inventory operations. In this setting, the contributions of our work are (i) the analytical derivation of the operating characteristics of the supply chain under consideration where inventory replenishment are regulated either with a continuous review $(Q, S)$ policy or a periodic review $(\mathbf{R}, T)$ policy; (ii) characterization of the parameter ranges where the performance of the periodic review policy, which is commonly adopted in practice due to ease-of-implementation, is fairly comparable to the continuous review policy; (iii) identifying the problem parameter settings where outsourcing should be the preferred option over in-house management of the transportation operations; and (iv) derivation of the steady state inventory position and inventory level probabilities as well as the service level expressions for the well-known $(Q, S)$ policy.

Our findings indicate that when optimal number of trucks are reserved in 3PL contracts and if the excess utilization charge is less than 25\%, 3PL contracts become more beneficial even if outsourcing cost is $25 \%$ more than in-house fleet costs under the selected parameter setting. Similarly, we provide numerical examples to illustrate the cost of 'practicality' in using a periodic review policy rather than a continuous review one under selected parameter settings. We observe that the relative performance of the $(\mathbf{R}, T)$ policy depends on the "size" of the supply chain and the truck sizes. In particular, for a small supply chain with one warehouse and two retailers, the relative cost of using $(\mathbf{R}, T)$ policy rather than a $(Q, S)$ policy ranges between 11.7-18.4\% for low capacity trucks and between 3.8-14.2\% for high capacity trucks. However, when the cost components are considered separately, it is observed that, for inventory holding, backordering, and extra truck usage costs, there are cases where $(\mathbf{R}, T)$ yields lower costs. Similarly, for a large supply chain with one warehouse and 16 retailers, the relative cost of using $(\mathbf{R}, T)$ policy rather than a $(Q, S)$ policy ranges between $3.2-11.0 \%$ for low capacity trucks and between $-1.6-$ $5.3 \%$ for high capacity trucks. $(\mathbf{R}, T)$ policy performs best in large supply chains with relatively higher truck capacity with respect to the lead time demand and gets more appealing as the number of retailers increases.

There are some limitations of our model, which might hint some future research directions. In our current model we assume that the transportation lead time is constant. In practice, however, the transit times might be stochastic not only because of the natural variability in transit times but also due to possible uncertainty in the availability time of the trucks after they are requested by the supply chain authority. If the transportation lead time is random with relatively small 
variability, a constant lead time assumption may still provide reliable results. However if the variability is not negligible, alternative modeling approaches can be employed. Specifically, in that case the appropriate model in the transportation base will be an $E_{Q} / G / \infty$ queue, for which results are available regarding the average queue lengths, waiting times etc. However in that setting, more complicated issues would arise in the inventory system since random transportation times will result in order crossings which should be addressed with suitable policies. Developing such integrated models and analyzing the impact of variability on the system performance would be a very interesting future topic for research. Two other underlying assumptions in our work are that the truck capacities are fixed and the warehouse is an ample supplier. Relaxing these assumptions would lead to a number of interesting problems from fleet size and type selection to rationing policies in dispatching, each of which merits further research effort. Our present work is predicated on the expectations of the system wide performance measures which imply that the decision maker is risk-neutral. In recent years however, most of the classical problems are revisited in order to take risk considerations into account. Similarly in our problem setting, the preferences of risk averse and risk seeking decision makers can be accounted for by using various alternative objective functions that incorporate risk considerations. To that end, developing the probability distributions of the output performance measures would also be a challenging future work.

Our study extends the work of Buyukkaramikli et al. (2014) when the transportation operations are outsourced, in an integrated in-house transportation and inventory management model under continuous review. An interesting venue for future research in this direction is to complement these studies by considering inventory models with in-house transportation under periodic review, possibly with alternative replenishment policies and contractual settings.

\section{Appendix}

Proof of Theorem 1. Suppose the system is at states $(n, i)$ and $(b, w)$ at an arbitrary time $t$ and at time $t+D$, respectively. This means that $n$ vehicles are busy and $i$ units of retail demands have been accumulated since the last order at time $t$; and similarly, $b$ vehicles are busy and $w$ units of retail demands have been accumulated since the last order at time $t+D$. Let $X$ denote the total demand observed between $t$ and $t+D$. Note that all busy trucks at time $t$ (i.e. $n$ trucks) will become available by time $t+D$. Therefore, in order to have $b$ trucks busy at time $t+D$, we must have $b Q+w=X+i$ and hence $X=b Q+w-i$. Consequently, we have the following relation:

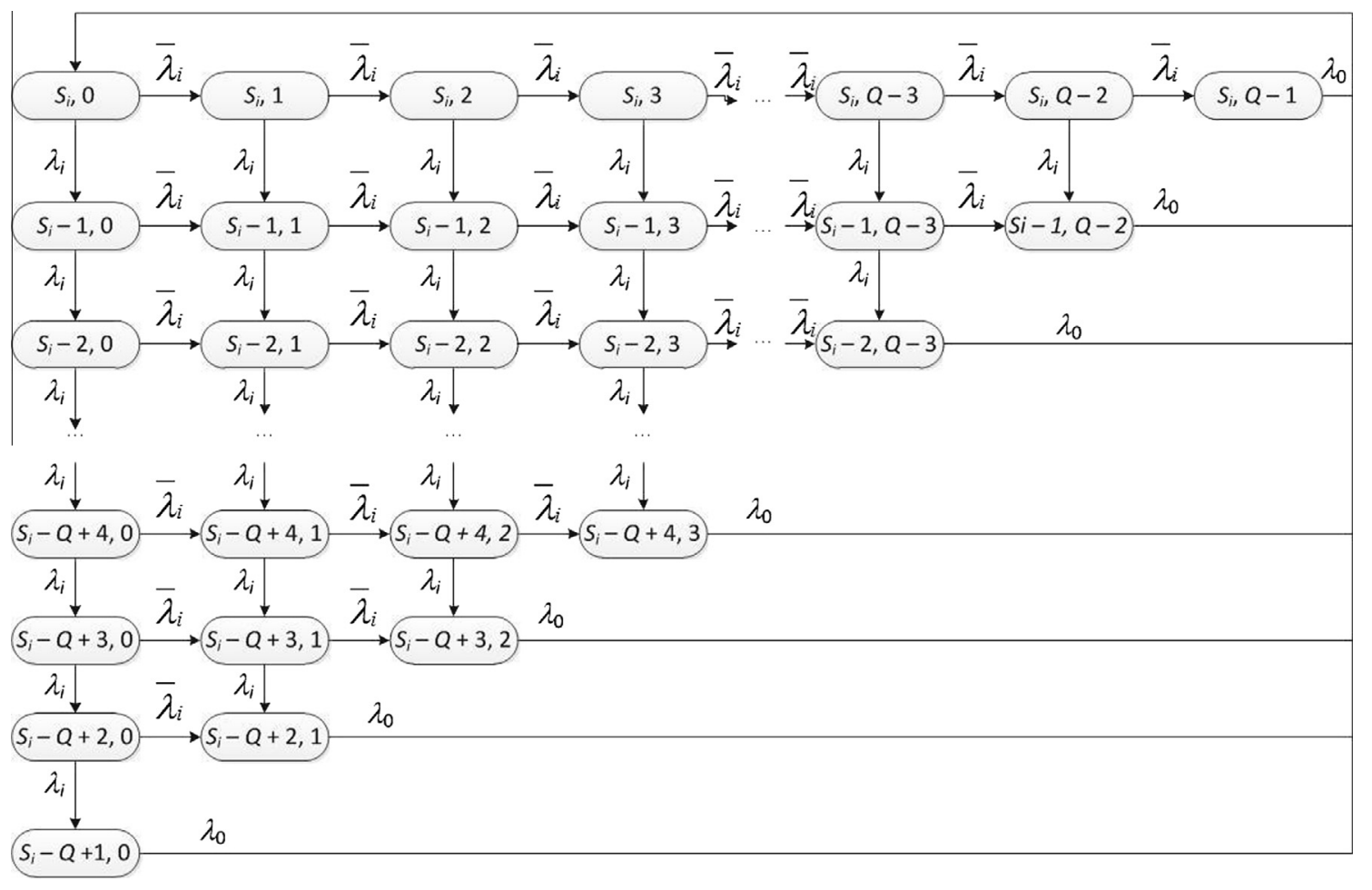

Fig. 5. State transition diagram for retailer $i . \bar{\lambda}_{i}=\lambda_{0}-\lambda_{i}$. 


$$
P_{t+D}\{(b, w)\}= \begin{cases}\sum_{n=0}^{\infty} \sum_{i=0}^{w} P_{t}\{(n, i)\} F_{P}\left(w-i \mid \lambda_{0} D\right) & \text { for } b=0,0 \leqslant w \leqslant Q-1, \\ \sum_{n=0}^{\infty} \sum_{i=0}^{Q-1} P_{t}\{(n, i)\} F_{P}\left(b Q+w-i \mid \lambda_{0} D\right) & \text { for } b \geqslant 1,0 \leqslant w \leqslant Q-1 .\end{cases}
$$

Changing the order of summations, we obtain the following relation:

$$
P_{t+D}\{(b, w)\}= \begin{cases}\sum_{i=0}^{w} P_{t}\{W=i\} F_{P}\left(w-i \mid \lambda_{0} D\right) & \text { for } b=0,0 \leqslant w \leqslant Q-1, \\ \sum_{i=0}^{Q-1} P_{t}\{W=i\} F_{P}\left(b Q+w-i \mid \lambda_{0} D\right) & \text { for } b \geqslant 1,0 \leqslant w \leqslant Q-1 .\end{cases}
$$

The resulting expression in the theorem follows from the fact that as $t \rightarrow \infty, P_{t}\{W=i\} \rightarrow \frac{1}{Q}$.

Proof of Theorem 2. In (1), the cost parameters that are dependent on $K$ are: $\phi(K)+\beta \frac{\phi(K)}{K} E\left[B_{q s}^{K+}\right]=a K\left(K+E\left[B_{q s}^{K+}\right]\right)$. Therefore in order to show the convexity of (1) in $K$, it is sufficient to show that $E\left[B_{q s}^{K+}\right]$ is convex in $K$. This result follows from the fact that $E\left[B_{q s}^{K+}\right]=E\left[(B-K)^{+}\right],(b-K)^{+}$is convex in $K$ for every fixed $b$, and expectation operator preserves convexity.

Proof of Theorem 3. Let $I P_{i}(t)=S_{i}-K(t)$ be the inventory position of the retailer $i$ at time $t$. We first derive the steady state probabilities $\pi_{S_{i}-k, j}=\lim _{t \rightarrow \infty} P\left(I P_{i}(t)=S_{i}-k, J(t)=j\right)$. The transition diagram of the $\{K(t), J(t), t \geqslant 0\}$ process is given in Fig. 5 .

Observe that the states of this process can be arranged as a $Q \times Q$ upper diagonal matrix where rows correspond to $S_{i}-k, k=0, \ldots, Q-1$ and columns correspond to the states $J(t)=j, j=0, \ldots, Q-1$. The $k$ th sub-diagonal from $k$ th row to $k$ th column has $k+1$ states on it, where each state $(S-m, j)$ satisfies $m+j=k$. For a clear exposition, we write below the balance equations for the states on the $k$ th sub-diagonal in the sequence $k=0,1, \ldots, Q-1$.

$$
\begin{aligned}
k=0: \quad \lambda_{0} \pi_{S_{i}, 0} & =\lambda_{0} \pi_{S_{i}-Q+1,0}+\lambda_{0} \pi_{S_{i}-Q+2,1}+\cdots+\lambda_{0} \pi_{S-1, Q-2}+\lambda_{0} \pi_{S, Q-1} \\
k=1: \quad \lambda_{0} \pi_{S_{i}-1,0} & =\lambda_{i} \pi_{S_{i}, 0} \\
\lambda_{0} \pi_{S_{1}, 1} & =\lambda_{i} \pi_{S_{i}, 0} \\
k=2: \quad \lambda_{0} \pi_{S_{i}-2,0} & =\lambda_{i} \pi_{S_{i}-1,0} \\
\lambda_{0} \pi_{S_{i}-1,1} & =\lambda_{i} \pi_{S_{i}-1,0}+\lambda_{i} \pi_{S_{i}, 1} \\
\lambda_{0} \pi_{S_{i}, 2} & =\lambda_{i} \pi_{S_{i}, 1} \\
\vdots & \vdots \\
k=Q-2: \quad \lambda_{0} \pi_{S_{i}-Q+2,0} & =\lambda_{i} \pi_{S_{i}-Q+3,0} \\
\lambda_{0} \pi_{S_{i}-Q+3,1} & =\lambda_{i} \pi_{S_{i}-Q+3,0}+\lambda_{i} \pi_{S_{i}-Q+4,1} \\
& \vdots \\
\lambda_{0} \pi_{S_{i}-1, Q-3} & =\bar{\lambda}_{i} \pi_{S_{i}-2, Q-3}+\lambda_{i} \pi_{S_{i}, Q-3} \\
\lambda_{0} \pi_{S_{i}, Q-2} & =\bar{\lambda}_{i} \pi_{S_{i}, Q-3} \\
\lambda_{0} \pi_{S_{i}-Q+1,0} & =\lambda_{i} \pi_{S_{i}-Q+2,0} \\
\lambda_{0} \pi_{S_{i}-Q+2,1} & =\bar{i}_{i} \pi_{S_{i}-Q+2,0}+\lambda_{i} \pi_{S_{i}-Q+3,1} \\
& \vdots \\
\lambda_{0} \pi_{S_{i}-1, Q-2} & =\bar{\lambda}_{i} \pi_{S_{i}-1, Q-3}+\lambda_{i} \pi_{S_{i}, Q-2} \\
\lambda_{0} \pi_{S_{i}, Q-1} & =\bar{\lambda}_{i} \pi_{S_{i}, Q-2}
\end{aligned}
$$

Let $\rho=\lambda_{i} / \lambda_{0}$. Then, $1-\rho=\bar{\lambda}_{i} / \lambda_{0}$. For any $k+j \in\{1,2, \ldots, Q-1$. $\}$, we will show by induction that

$$
\pi_{s_{i}-k, j}=\left(\begin{array}{c}
k+j \\
j
\end{array}\right) \rho^{t-j}(1-\rho)^{j} \pi_{s_{i}, 0}
$$

For $k=j=0$ and Eq. (3) is verified since it yields $\pi_{s_{i}, 0}=\pi_{s_{i}, 0}$. For $k+j=1$ we have $\pi_{S_{i}-1,0}=\rho \pi_{s_{i}, 0}$ and for $k=0, j=1, \pi_{s_{i}, 1}=(1-\rho) \pi_{s_{i}, 0}$, both of which satisfy (3). Now suppose that Eq. (3) satisfies all balance equations of $k+j=2,3, \ldots, r$.

For $k+j=r+1, j=0$ :

$$
\begin{aligned}
\lambda_{0} \pi_{S_{i}-(r+1), 0} & =\lambda_{i} \pi_{S_{i}-r, 0} \Rightarrow \pi_{S_{i}-(r+1), 0}=\rho \pi_{S_{i}-r, 0}=\rho\left(\begin{array}{l}
r \\
0
\end{array}\right) \rho^{r}(1-\rho)^{0} \\
& =\left(\begin{array}{c}
r+1 \\
0
\end{array}\right) \rho^{r+1}(1-\rho)^{0} \pi_{S_{i}, 0}
\end{aligned}
$$

For $t=r+1, j \in\{1,2, \ldots, r\}$ : 


$$
\begin{aligned}
\lambda_{0} \pi_{S_{i}-(r+1-j) j} & =\bar{\lambda}_{i} \pi_{S_{i}-(r+1-j, j-1)}+\lambda_{0} \pi_{S_{i}-(r-j), j} \\
\Rightarrow \pi_{S_{i}-(r+1-j), j} & =(1-\rho)\left(\begin{array}{c}
r \\
j-1
\end{array}\right) \rho^{r-(j-1)}(1-\rho)^{j-1} \pi_{S_{i}, 0}+\rho\left(\begin{array}{c}
r \\
j
\end{array}\right) \rho^{r-j}(1-\rho)^{j} \pi_{S_{i}, 0} \\
& =\left(\begin{array}{c}
r+1 \\
j
\end{array}\right) \rho^{r+1-j}(1-\rho)^{j} \pi_{S_{i}, 0}
\end{aligned}
$$

For $k+j=r+1, j=r+1$ :

$$
\lambda_{0} \pi_{S_{i}, r+1}=\bar{\lambda}_{i} \pi_{S_{i}, r} \Rightarrow \pi_{S_{i}, r+1}=(1-\rho)\left(\begin{array}{c}
r \\
r
\end{array}\right) \rho^{0}(1-\rho)^{r} \pi_{S_{i}, 0}=\left(\begin{array}{c}
r+1 \\
r+1
\end{array}\right) \rho^{0}(1-\rho)^{r+1} \pi_{S_{i}, 0} .
$$

Hence, we have verified (3). We next obtain the value of $\pi_{S_{i}, 0}$ from the boundary condition, as the $\pi$ values should add up to unity to result in

$$
\begin{aligned}
& \sum_{m=0}^{Q-1} \sum_{j=0}^{m}\left(\begin{array}{c}
m \\
j
\end{array}\right) \rho^{m-j}(1-\rho)^{j} \pi_{S_{i}, 0}=1 \\
& \pi_{S_{i}, 0} \sum_{t=0}^{Q-1} 1=1 \\
& \pi_{S_{i}, 0}=\frac{1}{Q}
\end{aligned}
$$

which implies

$$
\pi_{S_{i}-k, j}=\frac{1}{Q}\left(\begin{array}{c}
k+j \\
j
\end{array}\right) \rho^{k}(1-\rho)^{j} .
$$

Finally, noting that the marginal probability of the inventory position of the ith retailer is obtained as $P\left\{I P_{i}=S_{i}-k\right\}=\sum_{j=0}^{k} \pi_{S_{i}-k, j}$, which completes the proof.

Proof of Theorem 4. Suppose that at any given time $t$, the inventory position of retailer $i$ is $S_{i}-k$. Then, at time $t+L_{i}$ all outstanding orders must have arrived. Hence, if the inventory level at time $t+L_{i}$ is $x$ then we must have observed $S_{i}-k-x$ units of demand at retailer $i$ during $\left[t, t+L_{i}\right)$. Then we condition on each possible value of the inventory position at time $t$ to obtain the desired result for any inventory level $x$ at an arbitrary time $t+L_{i}$.

Proof of Theorem 5. Consider an arbitrary review period $k$. All trucks shipped at time $k$ will be busy until $k+D \leqslant k+(\eta+1) T$ where $\eta=\left\lfloor\frac{D}{T}\right\rfloor$ (see Fig. 6). All trucks that are shipped at review periods $k+T, k+2 T, \ldots, k+\eta T$ will be busy during the review cycle $[k+\eta T, k+(\eta+1) T]$. Hence $B_{r t}(\eta+1)$ trucks will be busy during $[k+\eta T, k+D]$, i.e. for a duration of $D-\eta T$ time units and $B_{r t}(\eta)$ trucks will be busy during $[k+D, k+(\eta+1) T]$, i.e. for a duration of $(\eta+1) T-D$ time units. Since each review cycle is identical to each other, the weighted average of these values in any period will yield the desired result.

Proof of Theorem 6. Let $\delta_{A C(K)}=A C(\mathbf{R}, T, K)-A C(\mathbf{R}, T, K-1)$ for $K=1,2,3 \ldots$ which is equal to

$$
\phi(K)+E\left[B_{r t}^{K+}\right]-\left(\phi(K-1)+E\left[B_{r t}^{(K-1)+}\right]\right)
$$

where $\phi(K)=a K$. Then,

$$
\delta_{A C(K)}=a+\left(E\left[B_{r t}^{K+}\right]-E\left[B_{r t}^{(K-1)+}\right]\right) .
$$

Moreover,

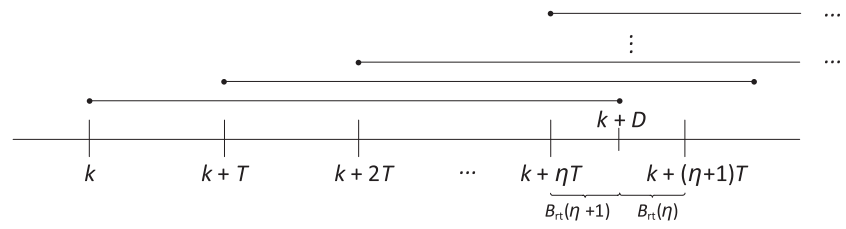

Fig. 6. Number of trucks busy. 


$$
\begin{aligned}
E\left[B_{r t}^{K+}\right]-E\left[B_{r t}^{(K-1)+}\right]= & \frac{E\left[\left(B_{r t}(\eta+1)-K\right)^{+}\right](D-\eta T)+E\left[\left(B_{r t}(\eta)-K\right)^{+}\right]((\eta+1) T-D)}{T} \\
& -\frac{E\left[\left(B_{r t}(\eta+1)-(K-1)\right)^{+}\right](D-\eta T)+E\left[\left(B_{r t}(\eta)-(K-1)\right)^{+}\right]((\eta+1) T-D)}{T} \\
= & \frac{(D-\eta T)(\Theta(K, \eta+1)-\Theta(K-1, \eta+1))+((\eta+1) T-D)(\Theta(K, \eta)-\Theta(K-1, \eta))}{T}
\end{aligned}
$$

where $\Theta(K, \eta)=E\left[\left(B_{r t}(\eta)-K\right)^{+}\right]$. After some algebra we obtain:

$$
\delta_{A C(K)}=a+\frac{(D-\eta T)\left(-\sum_{i=K}^{\infty} P\left(B_{r t}(\eta+1)=i\right)\right)+((\eta+1) T-D)\left(-\sum_{i=K}^{\infty} P\left(B_{r t}(\eta)=i\right)\right)}{T} .
$$

Note that $\delta_{A C(K)}$ increases as $K$ increases, hence total cost is convex with regards to $K$.

\section{References}

Alp, O., Erkip, N., Gullu, R., 2003. Optimal lot sizing/vehicle dispatching policies under stochastic lead times and stepwise fixed costs. Oper. Res. 51 (1), $160-$ 166.

Applegate, L., 2002. National Logistics Management. Harvard Business School, Case No: 9-801-110.

Archetti, C., Bertazzi, L., Paletta, G., Speranza, M.G., 2011. Analysis of the maximum level policy in a production-distribution system. Comput. Oper. Res. 38, $1731-1746$.

Atkins, D.R., Iyogun, P.O., 1988. Periodic versus can-order policies for coordinated multi-item inventory systems. Manage. Sci. 34, 791-796.

Berman, O., Wang, Q., 2006. Inbound logistic planning: minimizing transportation and inventory cost. Transp. Sci. 40 (3), 287-299.

Buyukkaramikli, N.C., Gurler, U., Alp, O., 2014. Coordinated logistics: joint replenishment with capacitated transportation for a supply chain. Prod. Oper. Manage. 23 (1), 110-126.

Burnson, P., 2012. 23rd Annual state of logistics report: slow and steady. <http://logisticsmgmt.com/images/site/LM1207_CovStateofLogistics_Rail.pdf> (accessed 10.12.2012).

Cachon, G., 2001. Managing a retailer's shelf space, inventory and transportation. Manuf. Serv. Oper. Manage. 3 (3), 211-229.

Cai, X., Chen, J., Xiao, Y., Xu, X., Yu, G., 2013. Fresh-product supply chain management with logistics outsourcing. Omega 41, 752-765.

Carbone, V., Stone, M.A., 2005. Growth and relational strategies used by the European logistics service providers: rationale and outcomes. Transp. Res. Part E 41 (6), 495-510.

Cheung, K.L., Lee, H., 2002. The inventory benefit of shipment coordination and stock rebalancing in a supply chain. Manage. Sci. 48 (2), 300-306.

Craumer, M., 2002. How to think strategically about outsourcing. Harvard Management Update May 2002, Article reprint no: U0205B.

Dullaert, W., Zamparini, L., 2013. The impact of lead time reliability in freight transport: a logistics assessment of transport economics findings. Transp. Res. Part E 49, 190-200.

EUbusiness, 2010. EU approves the acquisition of Giraud by French Geodis. <http://www.eubusiness.com/news-eu/mergers-giraud.157/> (accessed 7.7.2014).

FHWA, 2005. Logistics costs and US gross domestic product. Mhttp://ops.fhwa.dot.gov/freight/freight_analysis/econ_methods/lcdp_rep/> (accessed 10.12.2012).

Gurbuz, M.C., Moinzadeh, K., Zhou, Y.P., 2007. Coordinated replenishment strategies in inventory/distribution systems. Manage. Sci. 53 (2), $293-307$.

Hadley, G., Whitin, T.M., 1963. Analysis of Inventory Systems. Prentice Hall.

Kang, J-H., Kim, Y-D., 2010. Coordination of inventory and transportation managements in a two-level supply chain. Int. J. Prod. Econ. 123, 127-145.

Konur, D., Schaefer, B., 2014. Integrated inventory control and transportation decisions under carbon emissions regulations: LTL vs. TL carriers. Transp. Res. Part E 68, 14-38.

Kutanoglu, E., Lohiya, D., 2008. Integrated inventory and transportation mode selection: a service parts logistics system. Transp. Res. Part E 44, 665-683. Langley, J., Capgemini, 2013. Third party logistics study. <http://www.3plstudy.com/downloads/2013-3pl-study/> (accessed 1.10.2013).

Li, K., Sivakumar, A.I., Ganesan, V.K., 2008. Analysis and algorithms for coordinated scheduling of parallel machine manufacturing and 3PL transportation. Int. J. Prod. Econ. 115, 482-491.

Lieb, R., Bentz, B.A., 2005. The use of third-party logistics services by large American manufacturers: the 2004 survey. Transp. J. 44 (2), 5-15.

Lin, J., Yen, B., 2008. Highly Confident Transportation: Dynamics of IT Application in Supply Chain Management. Asia Case Res Center, Case No: HKU782. Madadi, A., Kurz, M.E., Ashayeri, J., 2010. Multi-level inventory management decisions with transportation cost consideration. Transp. Res. Part E 46, 719734.

Mutlu, F., Cetinkaya, S., 2010. An integrated model for stock replenishment and shipment scheduling under common carrier dispatch costs. Transp. Res. Part E. $46,844-854$.

Pantumsinchai, P., 1992. A comparison of three joint ordering policies. Decis. Sci. 23 (1), 111-127.

Selviaridis, K., Spring, M., 2007. Third party logistics: a literature review and research agenda. Int. J. Logist. Manage. 18 (1), $125-150$.

Tanrikulu, M.M., Sen, A., Alp, O., 2010. A joint replenishment policy with individual control and constant size orders. Int. J. Prod. Res. 48 (14), $4253-4271$.

Ton, Z., Wheelwright, S.C., 2005. Exel plc - Supply Chain Management at Haus Mart. Harvard Business School, Case No: 9-605-080.

Ulku, M.A., Bookbinder, J.H., 2012. Optimal quoting of delivery time by a third party logistics provider: the impact of shipment consolidation and temporal pricing schemes. Eur. J. Oper. Res. 221, 110-117.

Vasiliauskas, A.V., Jakubauskas, G., 2007. Principle and benefits of third party logistics approach when managing logistics supply chain. Transport 22 (2), 6872.

Zhao, Q.H., Chen, S., Leung, S.C.H., Lai, K.K., 2010. Integration of inventory and transportation decisions in a logistics system. Transp. Res. Part E 46, 913-925. 